\title{
Microstructural Characteristics of InGaZnO Thin Film Using an Electrical Current Method
}

\author{
Yen-Ting Chen ${ }^{1}$, Fei-Yi Hung ${ }^{1, *}$, Shoou-Jinn Chang ${ }^{2}$, Truan-Sheng Lui ${ }^{1}$ and Li-Hui Chen ${ }^{1}$ \\ ${ }^{1}$ Department of Materials Science and Engineering, Institute of Nanotechnology and Microsystems Engineering, \\ Center for Micro/Nano Science and Technology, National Cheng Kung University, Tainan 701, Taiwan, R. O. China \\ ${ }^{2}$ Institute of Microelectronics \& Department of Electrical Engineering, National Cheng Kung University, \\ Tainan 701, Taiwan, R. O. China
}

This research studied the microstructural characteristics and electronic properties of IGZO1114 films (atomic ratio In : Ga : Zn : O = $1: 1: 1: 4)$ with different annealing conditions. The solid-state electrical current method was used in the IGZO/In films (In layer was a channel) and the interface effect on the electrical current mechanism was discussed. The experimental results show the effect of the annealing temperature was larger than that of the deposition oxygen flow rate for the film resistances. IGZO film which was annealed at $575 \mathrm{~K}$ was able to stabilize the composition of the matrix. The electrical current experiment at room temperature confirmed that the diffusion of the IGZO/In film occurred through an electric current induced crystallization (EIC). The In layer not only assisted the recrystallization behavior of the IGZO film, but also improved the electrical resistance. [doi:10.2320/matertrans.M2011312]

(Received October 7, 2011; Accepted January 6, 2012; Published March 25, 2012)

Keywords: InGaZnO, crystallization, electric current induced crystallization (EIC), electrical resistance

\section{Introduction}

InGaZnO (IGZO) is one of the most attention-getting oxide semiconductor materials, and it is also an excellent amorphous oxide semiconductor (AOS). ${ }^{1-3)}$ It has higher electron motility (more than $10 \mathrm{~cm}^{2} \mathrm{~V}^{-1} \mathrm{~s}^{-1}$ ) in the deposited conditions at room temperature and has applications for lowtemperature devices. ${ }^{3,4)}$ The IGZO system has been reported constantly, and most of the reports have focused on the relationship between the atomic ratio and device characteristics. $^{2-5)}$ Furthermore, many papers have discussed the annealing effects of IGZO thin films, but the depositing oxygen flow conditions affecting the film matrix have still not been examined. ${ }^{6}$ Notably, these papers reported that the chemical composition of the IGZO films is not stable, and there are still reliability problems in the interface zone of devices. ${ }^{7)}$

Both the composition and annealing temperature of IGZO thin films affect the film resistance and interface problems, ${ }^{5-7}$ ) so stable IGZO thin film characteristics were obtained by using the co-sputtering system to deposit thin films and the samples were annealed with different depositing oxygen flow rates. ${ }^{8,9)}$ In fact, most single thin film systems have better electrical properties after an annealing treatment at 675$875 \mathrm{~K}$ for $1-2 \mathrm{~h}$. For a double layer structure, high temperature annealing always decreases the reliability in the interface zone due to interface diffusion. ${ }^{7,9,10)}$ Electrical current testing is a low temperature process carried out in atmosphere. ${ }^{11)}$ Its mechanism is an electrical current induced crystallization $(\mathrm{EIC})^{12,13)}$ that has been largely ignored in the optoelectronic thin films until now. So, the present study performed electrical current experiments on In thin film to discuss the electrical current crystallization characteristics of the IGZO/In structure at low temperature (During electrical current testing, the surface temperature of the film was raised by joule heat and was $<455 \mathrm{~K}$ ).

*Corresponding author, E-mail: fyhung@mail.ncku.edu.tw
Based on the above, this study used IGZO1114 ${ }^{1-6)}$ (atom ratio of $\mathrm{In}: \mathrm{Ga}: \mathrm{Zn}: \mathrm{O}=1: 1: 1: 4)$ thin films to investigate the effects of both the annealing temperature and depositing oxygen flow rate. Then we analyzed the crystallization characteristics of the IGZO films and interface diffusion effects by an electrical current testing with IGZO/In thin films. For actual applications, the interface of $\mathrm{Au} / \mathrm{IGZO}$ in the $\mathrm{Au} / \mathrm{IGZO} / \mathrm{In}$ structure was also observed. The results not only provide reference for recent TFT devices, but also clarify the interface atomic behavior and the electrical current induced crystallization (EIC) mechanism.

\section{Experimental Procedure}

\subsection{Structural analysis of $\mathrm{InGaZnO}_{4}$ film}

$\mathrm{InGaZnO}_{4}$ (atom ratio In : $\mathrm{Ga}: \mathrm{Zn}: \mathrm{O}=1: 1: 1: 4$ ) thin films were deposited on the glass substrates using a co-sputter (ULVAC, Model ACS-4000-C3). The sputtering power was $100 \mathrm{~W}$ and the depositing operating pressure was $2 \mathrm{~Pa}$. For the sputter depositing duration, we maintained the Ar gas flow rate at $20 \mathrm{sccm}$ and controlled the $\mathrm{O}_{2}$ gas flow rate for $0,0.1,2$ and $5 \mathrm{sccm}$ individually. In addition, some samples were annealed in a vacuum at 475, 525 and $575 \mathrm{~K}$ for $1 \mathrm{~h}$ respectively. The resistance of the films was measured using Hall effect measurement before and after annealing. ${ }^{11)}$

According to the data of electric resistance, three samples A, B and C (A sample: $\mathrm{O}_{2}$ flow rate is $0 \mathrm{sccm}$; annealing temperature is $575 \mathrm{~K}$, B sample: $\mathrm{O}_{2}$ flow rate is $5 \mathrm{sccm}$; without annealing, $\mathrm{C}$ sample: $\mathrm{O}_{2}$ flow rate $5 \mathrm{sccm}$; annealing temperature is $575 \mathrm{~K}$ ) were subjected to $\mathrm{X}$-ray and electron spectroscopy for chemical analysis (ESCA) to analyze the phase composition and the depth composition of the IGZO thin films. The $\mathrm{Cu}-\mathrm{K} \alpha$ standard $(\lambda=1.5403 \mathrm{~nm})$ was used, the scanning angle was varied from 20 to $90^{\circ}$ and the scanning velocity was $2^{\circ} \cdot \mathrm{min}^{-1}$. In addition, Raman spectroscopy of sample A was carried out with laser wavelength $523 \mathrm{~nm}$ and scanning range 300 to $700 \mathrm{~cm}^{-1}$. 

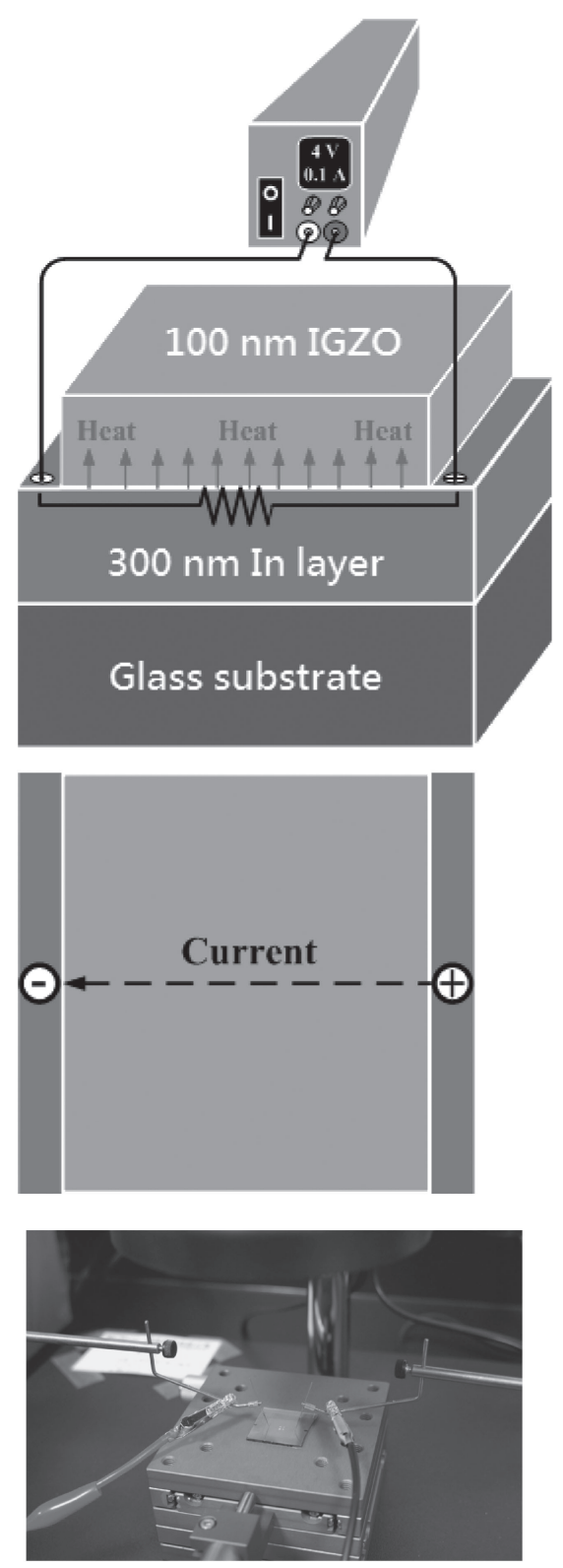

Fig. 1 Schematic illustration of electric current experiment for IGZO/In structure.

\subsection{Electric current test of InGaZnO/In structure}

The thickness of the Indium (In) conductive layer was about $300 \mathrm{~nm}$ on the glass substrates by sputtering. The sputtering parameters of the In film were $80 \mathrm{~W}$ for sputtering power, depositing Ar flow rate for $20 \mathrm{sccm}$ and operating pressure for $2 \mathrm{~Pa}$. Then $\mathrm{InGaZnO}_{4}$ film was deposited about $100 \mathrm{~nm}$ on the In conductive layer and finally fabricated an $\mathrm{InGaZnO}_{4} / \mathrm{In} /$ Glass thin film [The structure is shown in Fig. 1(a)].

The $\mathrm{IGZO}_{4}$ sputtering parameters were $100 \mathrm{~W}$ for sputtering power, depositing $\mathrm{O}_{2} / \mathrm{Ar}$ flow rate for $0 / 20 \mathrm{sccm}$ and operating pressure for $2 \mathrm{~Pa}$. Next, an electrical current test with a probe electrify meter [Fig. 1(b), the In film is an electric layer] was performed, and the voltage and current were within $2.0 \mathrm{~V}$ and $0.32 \mathrm{~A}$ in atmosphere (breakdown voltage of the In film was $2.8 \mathrm{~V}$ ). Finally, the electrical resistivity of the films before and after electrical current test was measured using the Hall effect measurement at room

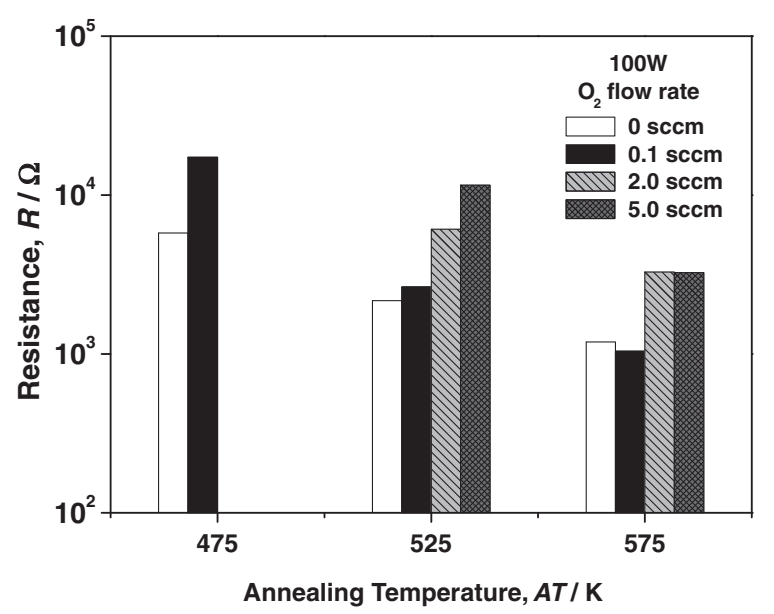

Fig. 2 Effect of IGZO1114 thin film resistance with different vacuum annealing temperature and oxygen flow rate.

temperature with indium ohmic contacts. Also, the diffusion behavior at the interface zones was examined using electron spectroscopy for chemical analysis (ESCA). For the Au/ $\mathrm{IGZO/In} \mathrm{structure,} \mathrm{the} \mathrm{thickness} \mathrm{of} \mathrm{the} \mathrm{Au} \mathrm{film} \mathrm{was} \mathrm{about}$ $50 \mathrm{~nm}$ and the interface mechanisms of $\mathrm{Au} / \mathrm{IGZO}$ were examined using high-resolution transmission electron microscopy (FE-TEM) and energy-dispersive X-rays (EDX).

\section{Result and Discussion}

\subsection{Microstructural characteristics of $\mathrm{InGaZnO}_{4}$ films}

Figure 2 shows the electric resistance of the $\mathrm{InGaZnO}_{4}$ films at the different temperatures of vacuum annealing and the deposited oxygen flow rate. The electric resistance of the film increases with increasing oxygen flow rate, but decreases as the annealing temperature rises. Also, the influence of the oxygen flow rate becomes smaller when the annealing temperature is higher. Both the annealing temperature and oxygen flow rate can control the electric resistance of the IGZO films effectively. Furthermore, the influence of annealing temperature is larger than the oxygen flow rate for the electric resistance of the film. For actual applications, the annealing temperatures are raised as far as possible $(<625 \mathrm{~K})$ to increase reliability and workability. So, it is not objective to declare that the electric resistance of the IGZO films was greatly affected by the depositing oxygen flow rate. ${ }^{9,10)}$ In fact, raising the annealing temperature not only improves the crystallization of the film but induces lower film electric resistance. Nevertheless, the TFT glass substrates will become softer and the interfaces of device undergo server diffusion when the annealing temperature is higher than $575 \mathrm{~K}$. For this reason, the IGZO films possess much applicability at $575 \mathrm{~K}$ vacuum annealing.

Figure 3 is the XRD pattern of the IGZO1114 films at several different conditions. It shows both the sputtering oxygen flow rate and the $575 \mathrm{~K}$ vacuum annealing can change the crystallization of the IGZO films. The crystal phase $\mathrm{InGaO}_{3}(\mathrm{ZnO})_{x}$ of low angle of the un-annealed film was found to be at $33^{\circ}$. After annealing, the matrix underwent a phase transform and induced the peak to shift from 33 to $24^{\circ}$. Therefore the two crystal phases caused the difference in 


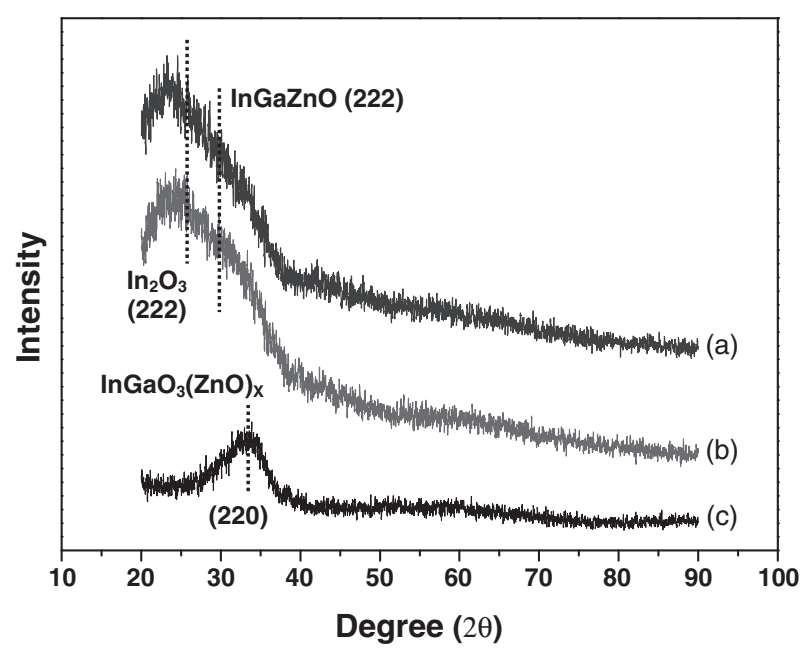

Fig. 3 XRD of IGZO1114 thin film: (a) $\mathrm{O}_{2} / 575 \mathrm{~K}$, (b) vacuum $/ 575 \mathrm{~K}$ and (c) $\mathrm{O}_{2}$ /un-annealed.

the electric resistance of the film, and the crystal phase $\left(\mathrm{IGZO}_{4}\right)$ of $24^{\circ}$ was able to decrease the electric resistance of the film. ${ }^{14)}$ After annealing, the matrix of IGZO was still partially amorphous and the XRD peak observed around $2 \theta=24^{\circ}$ was broader. In fact, some $\mathrm{InGaZnO}$ and $\mathrm{In}_{2} \mathrm{O}_{3}$ crystallized phases had exited. ${ }^{15-17)}$ Because both the depositing oxygen flow rate and crystallization can influence the oxygen vacancy defects and thus change the electric resistance of IGZO1114 films, the oxygen atom concentration and the atomic composition of depth in the matrix were detected using ESCA to estimate the effect of atomic ratios.

Figure 4 is ESCA analysis of the IGZO1114 film after annealing. The concentration of oxygen atoms was larger than the others. In addition, zinc had low adhesion on the deposited film and the concentration was lower. The concentration of the others (In; Ga) was similar. ${ }^{17,18)}$ The annealing without oxygen made the oxygen concentration lower in the matrix [Fig. 4(a)]. Notably, the In atom concentration increased when the film was deposited with oxygen and vacuum annealing was performed [Fig. 4(b)]. This explains why oxygen and the post annealing stabilized the composition of the matrix. This had a positive contribution on the optical characteristics of the IGZO1114 film.

Annealing conditions greatly influenced the properties of the IGZO1114 thin film. The relationship between the crystallization and the atom compositions was investigated by Raman spectroscopy. ${ }^{17,18)}$ The IGZO thin films were produced by the same process (deposited without oxygen) before and after $575 \mathrm{~K}$ vacuum annealing to perform Raman spectrum analysis. The results show that no Raman shift peak was found for the un-annealed IGZO thin film. On the other hand, a Raman spectrum of the annealed IGZO thin film was found and is shown in Fig. 5. Because there is no data about the IGZO Raman shift peak, the peak at Raman shift $466 \mathrm{~cm}^{-1}$ is defined as " $\mathrm{CH}$ peak". The values of the IGO and $\mathrm{ZnO}$ Raman shift peaks are also shown in Fig. 5 to compare with the data of the IGZO films. ${ }^{19,20)}$ So the $\mathrm{CH}$ peak is confirmed as not belonging to the IGO or $\mathrm{ZnO}$ systems. Compared to Figs. 2 and 3, low angle crystal phase $\left(24^{\circ}\right.$ : $\mathrm{IGZO}_{4}$ ) contributed to $\mathrm{CH}$ peak of Raman spectrum and affected the electric resistance of the film. (a)

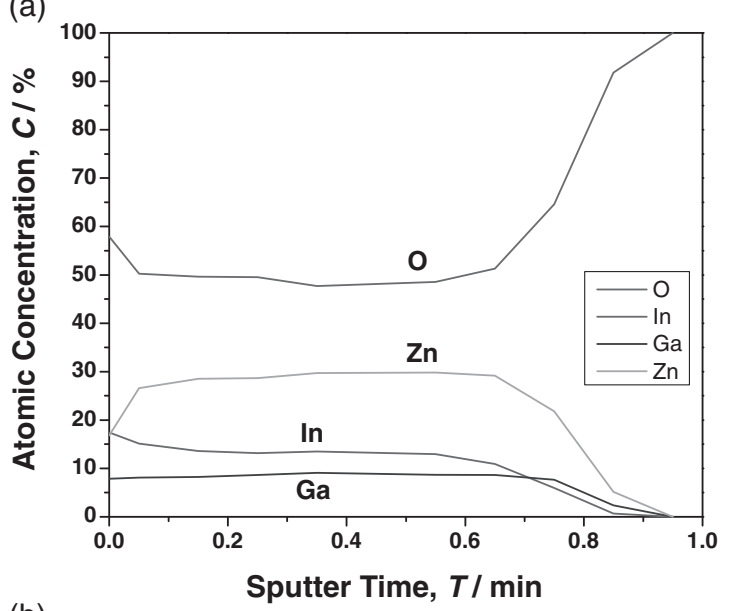

(b)

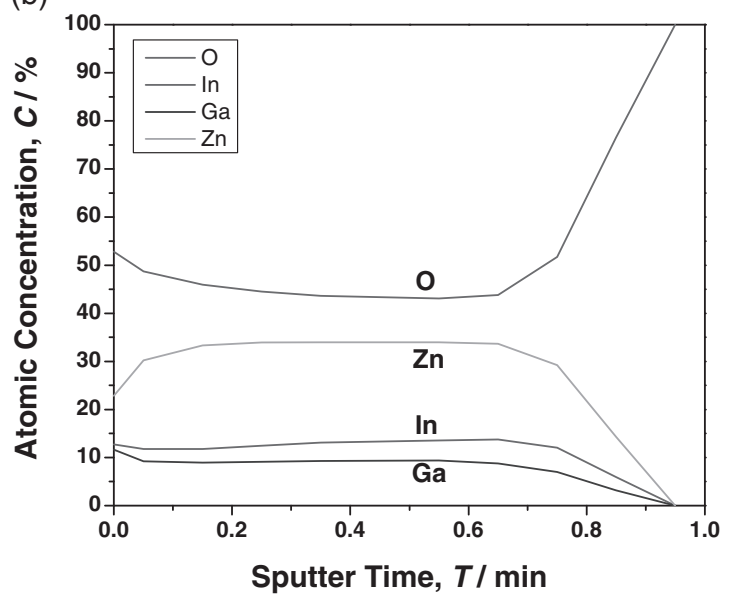

Fig. 4 ESCA profiles of IGZO1114 matrix after vacuum annealig at $575 \mathrm{~K}$ : (a) film deposited without oxygen and (b) film deposited with oxygen.

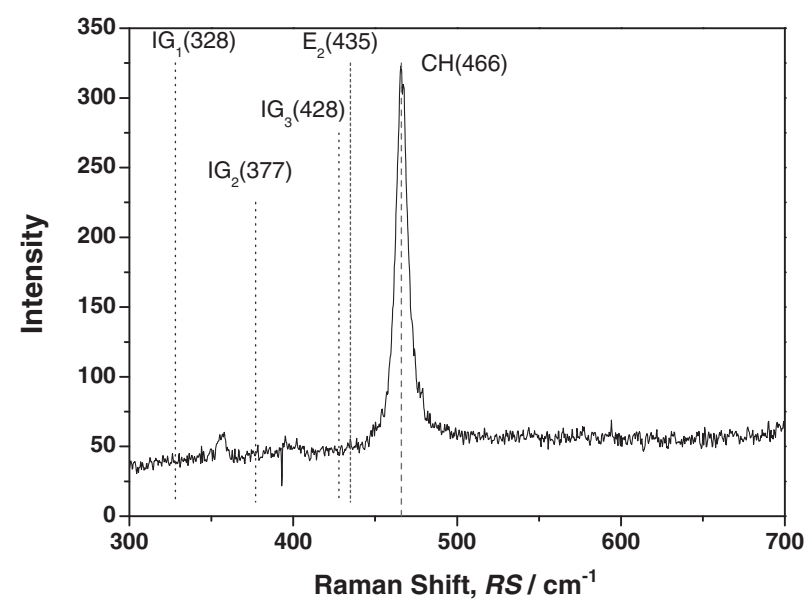

Fig. 5 Raman spectroscopy of $\mathrm{IGZO}_{4}$ after vacuum annealing at $575 \mathrm{~K}$. (IGO: $\mathrm{IG}_{1}, \mathrm{IG}_{2}, \mathrm{IG}_{3}$ and $\mathrm{ZnO}: \mathrm{E}_{2}$ )

\subsection{Electrical current induced crystallization of $\mathrm{InGaZnO}_{4} /$ Indium structure}

After annealing, the IGZO1114 thin films can obtain a stable phase structure, but the high temperature annealing process can easily induce interface diffusion (interface pollution) in devices and severely affect the workability. Electrical current induced crystallization (EIC) is a low 


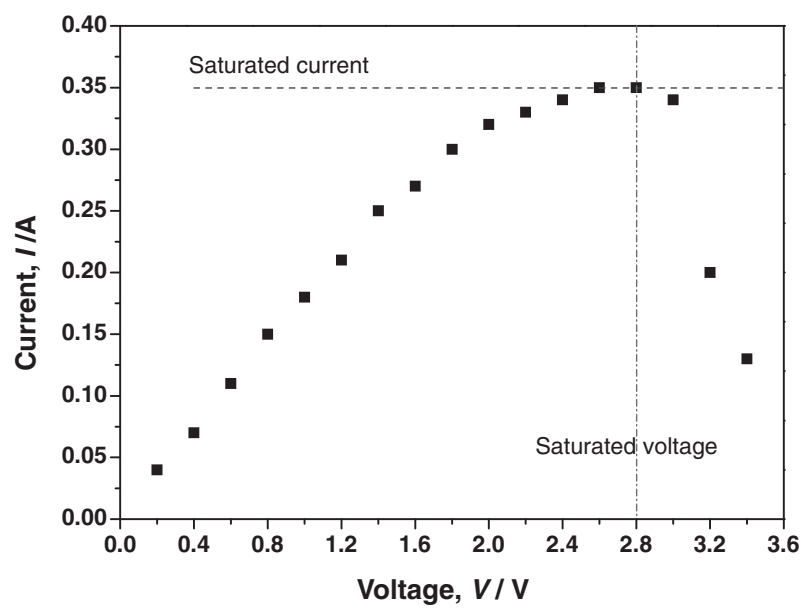

Fig. 6 The voltage (V)-current (I) curve of IGZO/In structures.

temperature process that can not only control the electric resistance of the films, but also avoids the problems of interface pollution can and even improve the crystallization efficiency in a short electrical current duration. ${ }^{11,21)}$

The present study chose Indium (In) material to be the conductive layer, and then deposited a IGZO1114 thin film to form an $\mathrm{InGaZnO}(100 \mathrm{~nm}) / \mathrm{In}(300 \mathrm{~nm}) /$ glass structure. A loading limit between the voltage and the current for EIC testing was measured. ${ }^{11-13,21)} \mathrm{We}$ controlled the conditions of EIC testing to obtain crystallization and diffusion doping by electron-migration and the joule heat, and then affected the electric resistance and the interface characteristics of the IGZO1114 thin films.

The EIC films will break if the input voltage or power is too large. So a power loading test of the un-annealed IGZO/ In thin film was performed. The voltage (V)-current (I) curve is shown in Fig. 6. It can be seen that the breakdown voltage of the In film is $2.8 \mathrm{~V}$, and the voltage of $2.0 \mathrm{~V}$ and the current of $0.32 \mathrm{~A}$ was selected for the EIC test. During $30 \mathrm{~min}$ of EIC testing (the EIC films were not damage), the surface temperature of the IGZO/In thin film was about $425 \mathrm{~K}$ by joule heat. Figure 7 shows that the EIC method decreased the electric resistance of the film at lower temperatures. ${ }^{9,10)}$ The joule heat of film was induced by EIC testing and raised the temperature. The heat source was in the In conductive layer, and the heat transfer direction was toward the upper IGZO film. Due to the interlayer (In film) being $300 \mathrm{~nm}$ thick, the induced joule heat $(\sim 425 \mathrm{~K})$ was insufficient to crystallize the as-deposited IGZO film. After EIC testing, the degree of crystallization had not only been raised but In had diffused into the as-deposited IGZO film to enhance the electrical properties. Meanwhile, electron-migration occurred in the In interlayer and the diffusion direction of the In ions was both parallel and perpendicular to the electron flow. This is why the IGZO matrix was saturated and higher doping concentrations were able to decrease the resistivity. Clearly, the In atoms provide a great contribution to diffusion doping under the EIC mechanism. ${ }^{11,21)}$

XPS depth profiles of the films before and after EIC test are shown in Fig. 8. Notably, the interface diffusion within the IGZO/In structure was confirmed. The border-top of this diffusion layer is where the In atom concentration curve

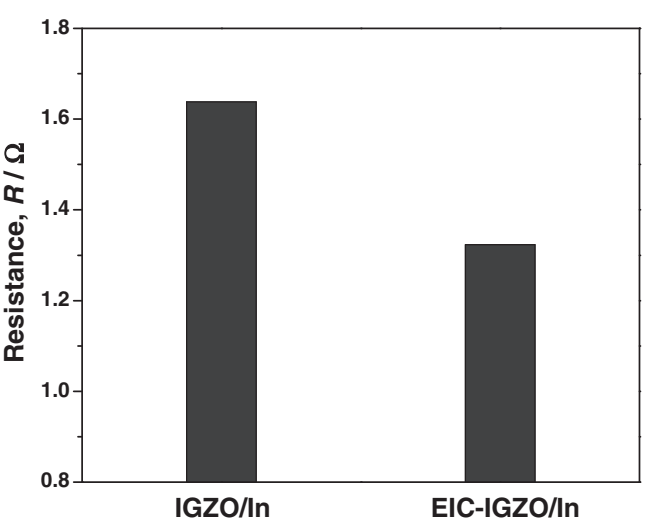

Fig. 7 Electric resistance of IGZO/In structures before and after EIC testing. The surface temperature of IGZO film was about $425 \mathrm{~K}$ by joule heat and electrical current time was $30 \mathrm{~min}$.
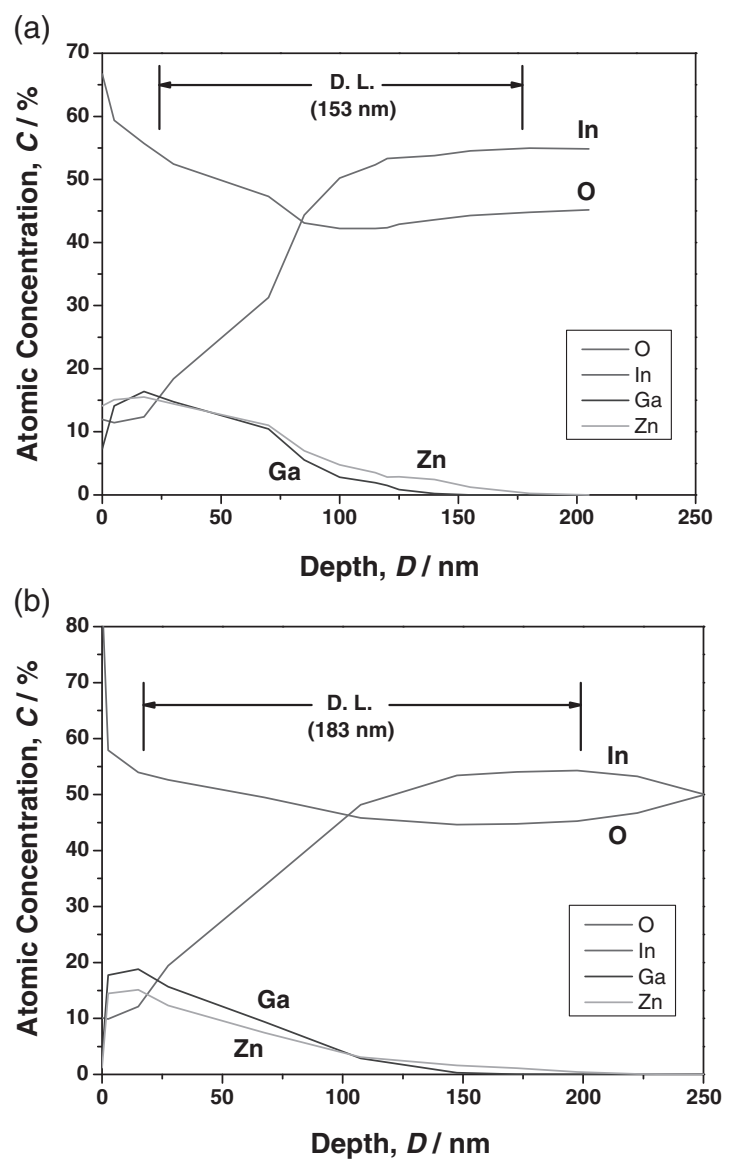

Fig. 8 ESCA depth profiles of IGZO/In films: (a) as-deposited and (b) after electrical current testing. (Diffusion Layer, D. L.)

begins rising and the border-bottom is where the $\mathrm{Zn}$ atom concentration was zero. After comparison, In atoms diffused into the IGZO matrix and $\mathrm{Zn}$, and Ga atoms diffused into the In layer occurring at the same duration. The thickness of the diffusion layer increased from 153 to $183 \mathrm{~nm}$ after EIC testing. In fact, there was an original diffusion layer before the EIC test (sputtering effect). This diffusion layer had grown top-ward and bottom-ward during EIC. Figure 9 shows the thickness profile of each layer for the EIC structure, and it also illustrates the interface effect (the IGZO 

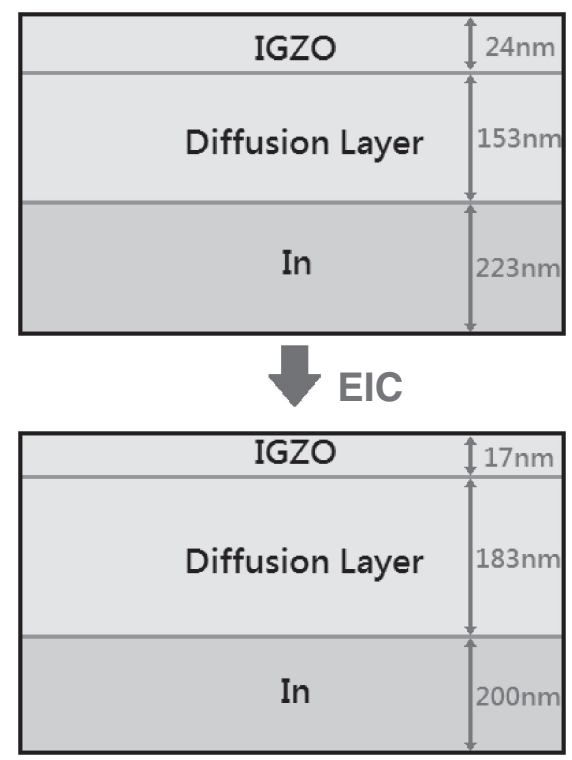

Fig. 9 Thickness profiles of each layer of EIC structure. The IGZO layer diminished when the diffusion layer grew.

\begin{tabular}{|c|}
\hline IGZO \\
\hline In \\
\hline Glass \\
\hline
\end{tabular}

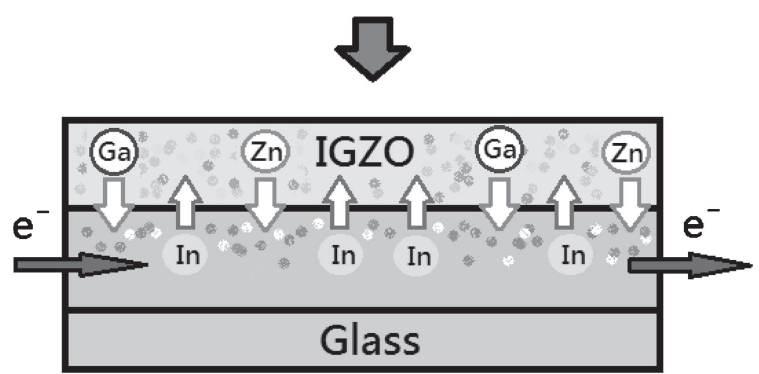

Fig. 10 The schematic illustration of electric current induced doping and recrystallization in $\mathrm{IGZO} / \mathrm{In}$ structure.

layer diminished when the diffusion layer grew) related to the electric resistance of the IGZO/In thin film after EIC testing. That is why the electric resistances of this IGZO/ In structure in Fig. 7 were lower than that of the un-EIC films.

According to the reference ${ }^{9,10)}$ and the above-mentioned results, the EIC mechanism is illustrated in Fig. 10. The electrical current induced diffusion in the interface between the In conductive layer and the IGZO film. When the diffusion layer grew, it not only improved the electric characteristics around the interface area, but also caused the partial crystallization in the IGZO film matrix. (When the In atomic concentration increased in the matrix, the rich $\operatorname{In}_{2} \mathrm{O}_{3}$ phase would increase.)

When the EIC testing time increased, the diffusion of the joule effect caused the diffusion layer to grow and also enhanced the doping effect in the IGZO film. Thus the

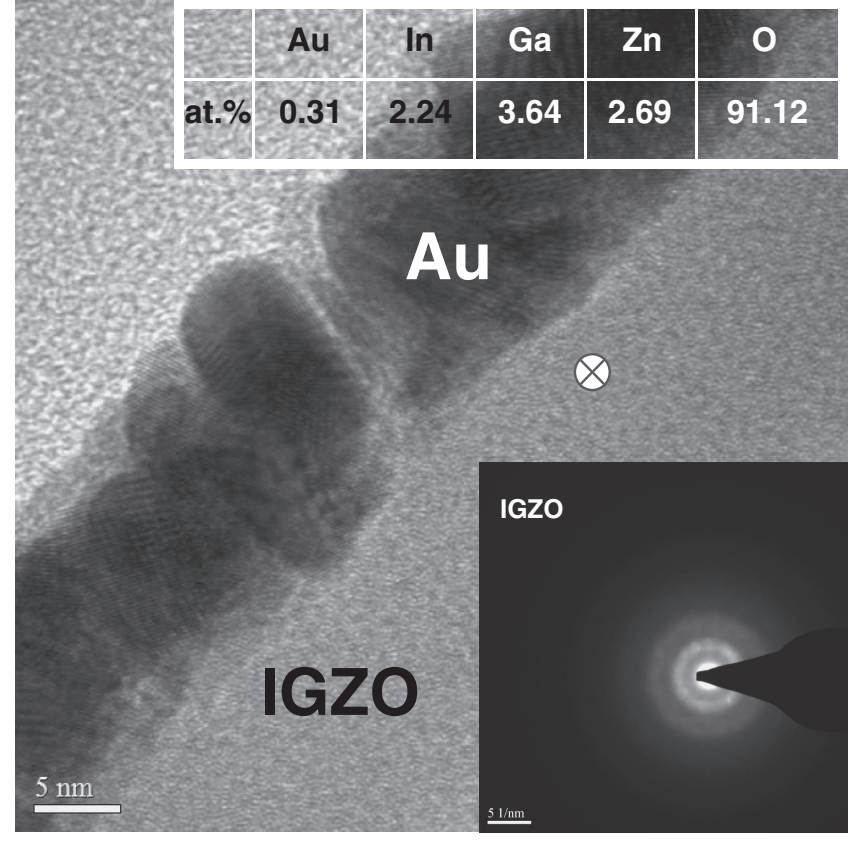

Fig. 11 Interface characteristics of $\mathrm{Au} / \mathrm{IGZO}$ after vacuum annealing at $575 \mathrm{~K}$. Au atoms had diffused into IGZO matrix (interface pollution).

electric characteristics of the IGZO/In structure have an improvement obviously due to the contribution from EIC induced In doping and crystallization in the IGZO matrix. For actual applications, the metal layer and IGZO matrix had interface diffusion after high temperature annealing. According to EDX results (Fig. 11), Au atoms were detected in the interface zone $(\mathrm{Au} / \mathrm{IGZO})$ and $\mathrm{Au}$ atoms diffused into the IGZO matrix. Due to the partial crystallization, low Au atoms could not affect the lattice of the matrix significantly. Notably, the $\mathrm{Au} / \mathrm{IGZO} / \mathrm{In}$ structure was fabricated for EIC testing and the Indium (In) was the conductive layer. After EIC testing, the interface between the Au film and IGZO film was also observed [Fig. 12(a)] and EDX was found [Fig. 12(b)] that $\mathrm{Au}$ atoms did not diffuse into the IGZO matrix (no interface pollution). ${ }^{15,22)}$ In other words, the EIC process caused the IGZO thin films to have the crystal conditions similar to the traditional annealing process, and moreover the structure had a heat diffusion and doping effect. This may provide a reference for the design of device structures.

\section{Conclusions}

The electric resistance of $\mathrm{InGaZnO}_{4}$ thin film increased with increasing the deposited oxygen flow rate, but raising the vacuum temperature reduced the electric resistance. After $575 \mathrm{~K}$ vacuum annealing, a thin film appeared the phase transform. Furthermore, the atomic concentration in the thin film matrix became stable and a $\mathrm{CH}$ peak of Raman shift $466 \mathrm{~cm}^{-1}$ was found after annealing.

EIC testing induced an interface effect of the IGZO/In thin film and made the diffusion layer grow. This not only improved the electric characteristics around the interface area, but also caused partial crystallization and heat diffusion doping in the IGZO films. 
(a)

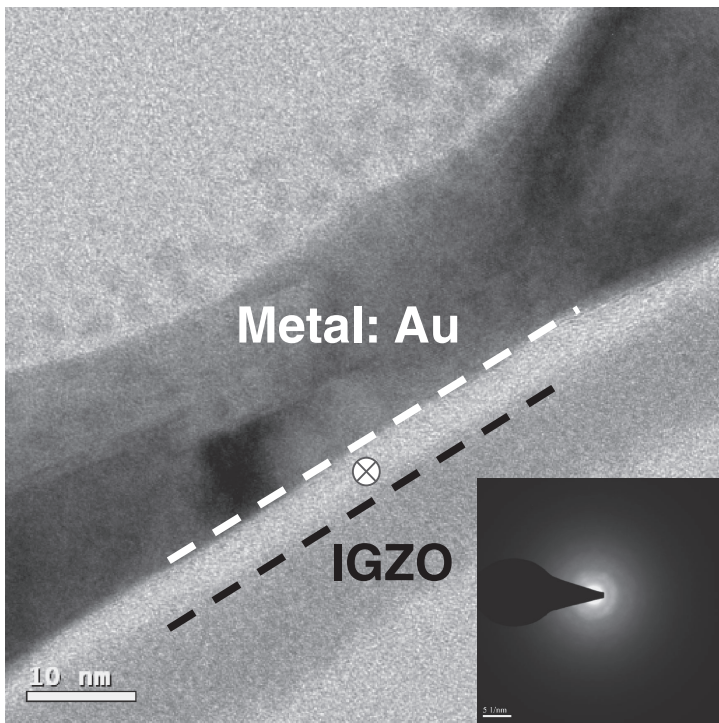

(b)

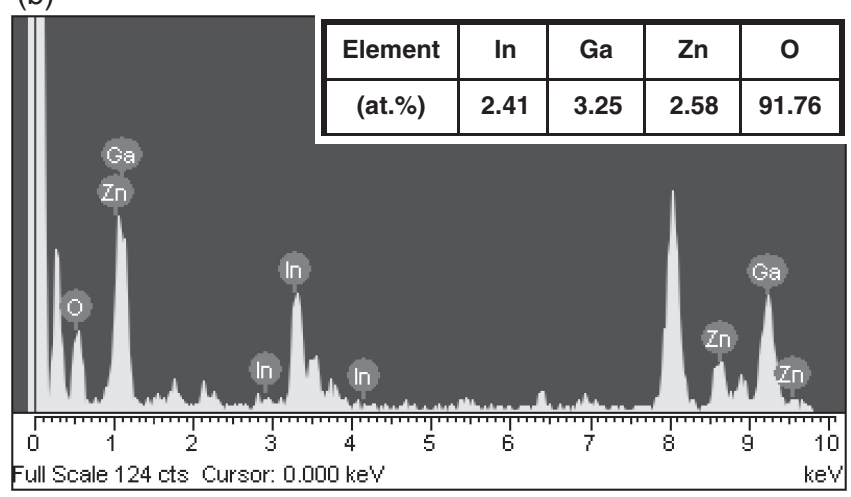

Fig. 12 Interface structure of $\mathrm{Au} / \mathrm{IGZO} / \mathrm{In}$ : (a) cross section image and (b) EDX of IGZO zone at the circle.

\section{Acknowledgements}

The authors are grateful to The Instrument Center of National Cheng Kung University, the Center for Micro/Nano
Science and Technology (D100-2700) and NSC 100-2221-E006-094; NSC100-2221-E-006-092 for the financial support.

\section{REFERENCES}

1) Y. K. Moon, S. Lee, D. H. Kim, D. H. Lee, C. O. Jeong and J. W. Park: JPN J. Appl. Phys. 48 (2009) 031301-1.

2) G. H. Kim, B. D. Ahn, H. S. Shin, W. H. Jeong, H. J. Kim and H. J. Kim: Appl. Phys. Lett. 94 (2009) 233501.

3) Y. Kikuchi, K. Nomura, H. Yanagi, T. Kamiya, M. Hirano and H. Hosono: Thin Solid Films 518 (2010) 3017.

4) M. Nakata, K. Takechi, T. Eguchi, E. Tokumitsu, H. Yamaguchi and S. Kaneko: JPN J. Appl. Phys. 48 (2009) 081607.

5) D. P. Gosain and T. Tanaka: JPN J. Appl. Phys. 48 (2009) 03 B018.

6) H. Godo, D. Kawae, S. Yoshitomi, T. Sasaki, S. Ito, H. Ohara, H. Kishida, M. Takahashi, A. Miyanaga and S. Yamazaki: JPN J. Appl. Phys. 49 (2010) 03CB04.

7) K. Takechi, M. Nakata, T. Eguchi, H. Yamaguchi and S. Kaneko: JPN J. Appl. Phys. 48 (2009) 011301.

8) J. Lee, J. S. Park, Y. S. Pyo, D. B. Lee, E. H. Kim, D. Stryakhilev, T. W. Kim, D. U. Jin and Y. G. Mo: Appl. Phys. Lett. 95 (2009) 123502.

9) S. Y. Lee, S. Chang and J. S. Lee: Thin Solid Films 518 (2010) 3030.

10) A. Sato, M. Shimada, K. Abe, R. Hayashi, H. Kumomi, K. Nomura, T. Kamiya, M. Hirano and H. Hosono: Thin Solid Films 518 (2009) 1309.

11) F. Y. Hung: Mater. Trans. 52 (2011) 1138-1141.

12) H. C. Chuang, F. Y. Hung, T. S. Lui and L. H. Chen: Mater. Trans. 52 (2011) 25-30.

13) F. Y. Hung, Z. R. Wang, T. S. Lui and L. H. Chen: Mater. Trans. 46 (2005) 1820-1824.

14) B. D. Ahn, H. S. Shin, G. H. Kim, J. S. Park and H. J. Kim: JPN J. Appl. Phys. 48 (2009) 03B019.

15) I. K. Jeong, H. L. Park and S. I. Mho: Solid State Commun. 108 (1998) 823-826.

16) Y. Sun and J. A. Rogers: Adv. Mater. 19 (2007) 1897-1916.

17) K. Inoue, K. Tominaga, T. Tsuduki, M. Mikawa and T. Moriga: Vacuum 83 (2009) 552-556.

18) J. A. Jeong and H. K. Kim: Thin Solid Films 519 (2011) 3276-3282.

19) J. H. Zheng, Q. Jiang and J. S. Lian: Appl. Surf. Sci. 257 (2011) 50835087.

20) N. Tripathi, K. Vijayarangamuthu and S. Rath: Mater. Chem. Phys. 126 (2011) 568-572.

21) K. J. Chen, F. Y. Hung, T. S. Lui, S. J. Chang and Z. S. Hu: Mater. Trans. 52 (2011) 1560-1564.

22) S. Hwang, J. H. Lee, C. H. Woo, J. Y. Lee and H. K. Cho: Thin Solid Films 519 (2011) 5146-5149. 